\title{
3D models related to the publication: Morphogenesis of the liver during the human embryonic period
}

\author{
Hirose Ayumi ${ }^{1}$, Nakashima Takashi ${ }^{1}$, Shiraki Naoto ${ }^{1}$, Yamada Shigehito ${ }^{1,2}$, Uwabe Chigako², Kose Katsumi ${ }^{3}$, \\ Takakuwa Tetsuya ${ }^{1 *}$ \\ ${ }^{1}$ Human Health Science, Graduate School of Medicine, Kyoto University, Sakyo-ku Shogoin Kawahara-cho 53, Kyoto, 606-8507, Japan \\ ${ }^{2}$ Congenital Anomaly Research Center, Graduate School of Medicine, Kyoto University, Sakyo-ku Yoshida-Konoe-cho Kyoto, 606-8501, Japan \\ 3 Institute of Applied Physics, University of Tsukuba, Tenoudai 1-1-1, Tsukuba, Ibaragi, 305-8573, Japan \\ ${ }^{*}$ Corresponding author: tez@hs.med.kyoto-u.ac.jp
}

\section{Abstract}

The present 3D Dataset contains the 3D models analyzed in: Hirose, A., Nakashima, T., Yamada, S., Uwabe, C., Kose, K., Takakuwa, T. 2012. Embryonic liver morphology and morphometry by magnetic resonance microscopic imaging. Anat Rec (Hoboken) 295, 51-59. doi: 10.1002/ar.21496

Keywords: human embryo, human liver, magnetic resonance imaging, three-dimensional reconstruction

Submitted:2016-02-15, published online:2016-03-17. doi: 10.18563/m3.1.4.e1

$$
\begin{aligned}
& 3 \\
& \frac{0}{2} \\
& \frac{3}{2} \\
& \frac{0}{2} \\
& 3 \\
& 2
\end{aligned}
$$

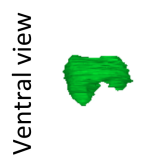

14

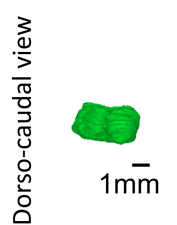

17
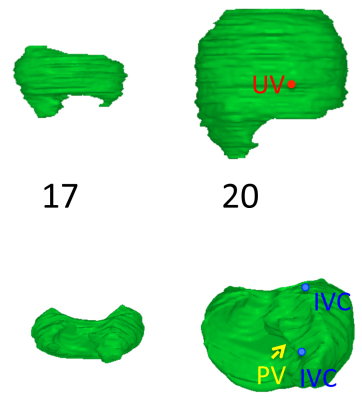

20

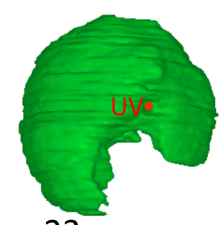

23

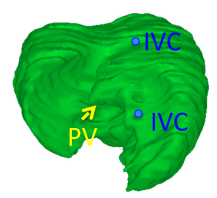

Figure 1. Representative images of the liver at Carnegie stage (CS) 14, CS 17, CS 20, and CS 23. IVC; inferior vena cava, PV; portal vein, UV; umbilical vein

\section{INTRODUCTION}

The morphogenesis of the human liver was visualized using images derived from human embryo specimens between Carnegie stage (CS) 14 and CS 23 from the Kyoto Collection, which were acquired with a magnetic resonance microscope equipped with a 2.35 -T superconducting magnet. (see Table $1)$.

\section{METHODS}

Well-preserved human embryos between Carnegie stage (CS) 14 and the CS 23 (approximately 5-8 weeks after fertilization) were selected from Kyoto Collection for MR microscopic imaging (Nishimura et al, 1968; Shiota et al, 2007; O'Rahilly $\&$ Müller, 1987). The MR images of the embryos were ac- quired using a super-parallel MR microscope developed with a $2.35 \mathrm{~T}$ horizontal bore $(40 \mathrm{~cm})$ superconducting magnet (Matsuda et al., 2007). MRI data from selected embryos were analyzed precisely as serial 2D and reconstructed 3D images. The structure of the stomach was reconstructed in all samples using OsiriX ${ }^{\mathrm{TM}}$ software version 3.7.1 (Pixmeo SARL, Geneva, Switzerland). The 3D surface models were then processed with ISE-MeshTools (Lebrun, 2014); each model was orientated and labelled using this software. All labels were provided in .flg format. The 3D surface models are also provided in .ply format, and can therefore be opened with a wider range of freeware. This study was approved by The Committee of Medical Ethics of Kyoto University Graduate School of Medicine, Kyoto, Japan (E986). Representative images of 3D models of livers are shown in Fig. 1.

\section{ACKNOWLEDGEMENTS}

This study was supported by Grant Nos. 25461642, 24119002, 26220004, 15H01119, 15K08134, 15H05270, 15H01121, and 15K15014 from the Japan Society for the Promotion of Science.

\section{BIBLIOGRAPHY}

Hirose, A., Nakashima, T., Yamada, S., Uwabe, C., Kose, K., Takakuwa, T. 2012. Embryonic liver morphology and morphometry by magnetic resonance microscopic imaging. Anat Rec (Hoboken) 295, 51-59. doi: 10.1002/ar.21496

Lebrun, R., 2014. ISE-MeshTools, a 3D interactive fossil reconstruction freeware. 12th Annual Meeting of EAVP, Torino, Italy. 


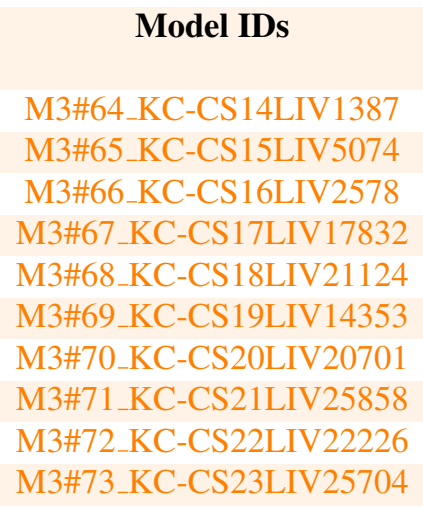

Taxon

Homo sapiens

Homo sapiens

Homo sapiens

Homo sapiens

Homo sapiens

Homo sapiens

Homo sapiens

Homo sapiens

Homo sapiens

Homo sapiens

Table 1. List of models
Developmental stage (Carnegie stage (CS))

CS 14

CS 15

CS 16

CS 17

CS 18

CS 19

CS 20

CS 21

CS 22

CS 23

Matsuda, Y., Ono, S., Otake, Y., Handa, S., Kose, K., Haishi, T., Yamada, S., Uwabe, C., Shiota, K., 2007. Imaging of a large collection of human embryo using a super-parallel MR microscope. Magn Reson Med Sci 6, 139-146. doi: 10.2463/mrms.6.139

Nishimura, H., Takano, K., Tanimura, T., Yasuda, M., 1968. Normal and abnormal development of human embryos: first report of the analysis of 1,213 intact embryos. Teratology 1 , 281-290. doi: 10.1002/tera.1420010306

O'Rahilly, R., Müller, F., 1987. Developmental stages in human embryos: including a revision of Streeter's Horizons and a survey of the Carnegie Collection. Washington, D.C.: Carnegie Institution of Washington.

Shiota, K., Yamada, S., Nakatsu-Komatsu, T., Uwabe, C., Kose, K., Matsuda, Y., Haishi, T., Mizuta, S., Matsuda, T., 2007. Visualization of human prenatal development by magnetic resonance imaging (MRI). Am J Med Genet A 143A, 3121-3126. doi: 10.1002/ajmg.a.31994 